\title{
Odometry Based on Auto-Calibrating Inertial Measurement Unit Attached to the Feet
}

\author{
Dinesh Atchuthan $^{1}$, Angel Santamaria-Navarro ${ }^{2}$, Nicolas Mansard $^{1}$, Olivier Stasse ${ }^{1}$, Joan Solà ${ }^{1,2}$
}

\begin{abstract}
Location of pedestrian in indoor environment remains an open problem. A cheap and reliable sensor in this context is the inertial measurement units (IMU), carried by the pedestrian while he/she is walking. However, due to the bias of both the accelerometer and the gyroscope, integrating directly the inertial measurements leads to tremendous drift, as the state of the system (position, orientation, velocity, bias) is not fully observable. In this paper, we consider the specific case where an IMU is attached to one of the pedestrian's feet. We exploit specific prior knowledges (i.e. the fact that the foot lands at zero velocity on a horizontal plane) in order to make the full state of the IMU observable. The inertial measurements and these prior knowledges are gathered in a graphical model (a factor graph), and are exploited to build a maximum-likelihood estimator. The technical difficulty is to handle the size of the graph such that it is tractable in a limited time window, that we do by relying on the pre-integration technique. In that existing framework, our contributions are to reformulate the pre-integration method using quaternions while giving a simpler algebraic formulation, and to apply this method for estimating the human foot-pose during walking. We validate these concepts on several longrange trajectories capture with human subject and compare the results with ground-truth measurements (coming from a motion capture system) and previous results of the state of the art.
\end{abstract}

\section{INTRODUCTION}

Context: Indoor person localization is an open challenge in various situations: location-based life improving services, firefighters localization and navigation, patients tracking motion monitoring, medical observation, accident monitoring, mobility and independence of partially-sighted or blind persons, etc. As GPS are not available indoor, and because relying on a network of fixed sensors (cameras, RFID) also raised many open questions, an appealing way to localize a body in space is to use odometry information measured by embedded inertial measurement units (IMUs). In this context, while performing the integration it is mandatory to take into account the IMU biases. As the biases vary with time and physical conditions, it must be estimated on-line while processing the measurements. Furthermore, it is desirable that additional information coming from other sensors can be integrated in the same estimation process. Similarly to the strategies adopted in simultaneous localization and mapping (SLAM), sparse measurements or additional information (e.g. coming from intermittent absolute localization, or from a sparse sensor network) would benefit to the localization process when available. With these requirements coming from the context in mind, we propose to define an estimator

\footnotetext{
1 CNRS - LAAS, Toulouse, France, $\{$ first.last $\}$ la las.fr

2 IRI, UPC, Barcelona, \{asantamaria, jsola\}@iri.upc.edu
}

based on graphical models, able to accurately and efficiently integrate inertial measurements while estimating the IMU biases. Thanks to the graphical model, the estimator will then be able to fuse additional measurements coming from other sensors or additional prior information provided by the application. The setup considered in this work is a pedestrian walking on structured terrain (flat floor or stairs) with an IMU attached to one of his or her feet.

Methodology: Graphical methods have been extensively used to implement such fusion strategies [1], [2]. They are well-suited to gather information from sensors and draw conclusions. The underlying principle is to consider that despite all the information gathered from the sensors, we still have uncertainties about the true state of the world due to imperfections of the sensors. Several states of the world can thus be considered as probable. Relying on probabilistic formulations is a way to find out the most probable one. Furthermore, graphical representation are able to accurately model complex estimation problems [3] in a versatile way.

Graphical models have been used for large modeling estimation problems by means of sparse networks of constraints and particularly in robotics where SLAM and visual odometry problems have reached a high degree of maturity in great part thanks to these tools. The graphical representation also allows for the design of powerful nonlinear estimation solvers, which can be built taking into account the needs for accuracy, robustness and computational performances.

In order to keep the problem tractable and maintain realtime performance, a key point is to prevent the graph from being too large given a time window. IMUs are challenging in this regard, as their high frequency measurement rate create large sets of data. Pre-integration of IMU measurements helps to reduce the size of the underlying graph by squeezing 100 to 1000 measures into a single pre-integrated Bayesian node [4]. Direct pre-integration leads to a dependency of the resulting node on the initial integration conditions, implying to integrate again (and again) when an optimizer process the graph. It was later suggested to make this pre-integrated data independent from the initial state from which it was computed [5]. Consequently, the IMU measurements can simply be disregarded even when the initial "pre-integration" conditions change while the numerical solver optimized the maximum-likelihood trajectory.

Contributions: In this paper, we follow a similar methodology. We define a graphical model where pre-integrated data are added at key-frame instants (about 2Hz), summarizing IMU measurements captured at about $1 \mathrm{kHz}$. The state that we want to estimate considers the position, orientation 
and linear velocity (dimension 9) of the IMU attached to the foot, along with the time-varying accelerometer and gyroscope biases (dimension 6). We also implement the prior knowledge that the foot lands horizontally, by adding Bayesian nodes when the foot lands and takes off. This prior knowledge make the considered state observable. We then use a numerical solver to maximize the likelihood of the measurements and prior knowledge along the past trajectory in a sliding window.

The first contribution of this paper is to reformulate the pre-integration method introduced in [5] using quaternion representation and to give a detailed and simpler algebraic derivation. The second contribution is to apply this method for estimating the human foot-pose during walking. To this end, we build a graphical model formulation of the problem by gathering information. Once the formulation is completed, a non-linear least squares optimizer is used to solve the problem and find the most probable solution in the leastsquare sense [6].

\section{GRAPH-BASED INERTIAL-KINEMATIC ODOMETRY}

\section{A. Quaternion rotations}

We define the quaternion-by-vector product $\odot$ so that

$$
\mathbf{q} \odot \mathbf{v} \triangleq \mathbf{q} \otimes \mathbf{v} \otimes \mathbf{q}^{*}
$$

where $\mathbf{q}^{*}$ denotes the dual quaternion of $\mathbf{q}$. The symbol $\otimes$ stands for the product of quaternions and $\odot$ corresponds to the quaternion-by-vector which performs a 3D rotation of an input vector $\mathbf{v}$. Notice that if $\mathbf{R}$ is the rotation matrix equivalent to the quaternion $\mathbf{q}$, then $\mathbf{q} \odot \mathbf{v}=\mathbf{R} \mathbf{v}$. This straightforward equivalence enables us to define all the forthcoming IMU pre-integration algebra in a way that allows a direct transcription between the $S^{3}$ and $S O(3)$ spaces of representation.

\section{B. Graph-based iterative optimization}

In graph-based optimization, the problem is represented as a graph, where the nodes refer to the variables, and the edges, called factors, represent the geometrical constraints that link between variables produced by the measurements. The state $\mathbf{x}$ is modeled as a multi-variate Gaussian distribution, and in our case it includes foot poses and velocities $(\mathbf{p}, \mathbf{q}, \mathbf{v})$ and IMU biases $\left(\mathbf{a}_{b}, \boldsymbol{\omega}_{b}\right)$ at selected keyframes along the trajectory (see Fig. 1). For each factor, we can define an error or a residual $\mathbf{r}$ as the discrepancy between a measurement $\mathbf{z}$ and its expectation given the involved state variables,

$$
\mathbf{r}(\mathbf{x})=h(\mathbf{x})+\mathbf{v}-\mathbf{z}, \quad \mathbf{v} \sim \mathcal{N}\left(0, \mathbf{\Omega}^{-1}\right)
$$

being $h(\mathbf{x})$ the sensor measurement model and $\boldsymbol{\Omega}$ the information matrix of the measurement Gaussian noise $\mathbf{v}$. Importantly, the functions $h(\mathbf{x})$ and $\mathbf{r}(\mathbf{x})$ are very sparse, since only a small handful of blocks of $\mathbf{x}$ are involved in each factor, which results in a loosely connected graph. In case of variables defined on manifolds, such as quaternions or rotation matrices, we must rewrite (2) as $\mathbf{r}(\mathbf{x})=(h(\mathbf{x}) \oplus \mathbf{v}) \ominus \mathbf{z}$. The $\oplus$ and $\ominus$ symbols correspond to the addition and

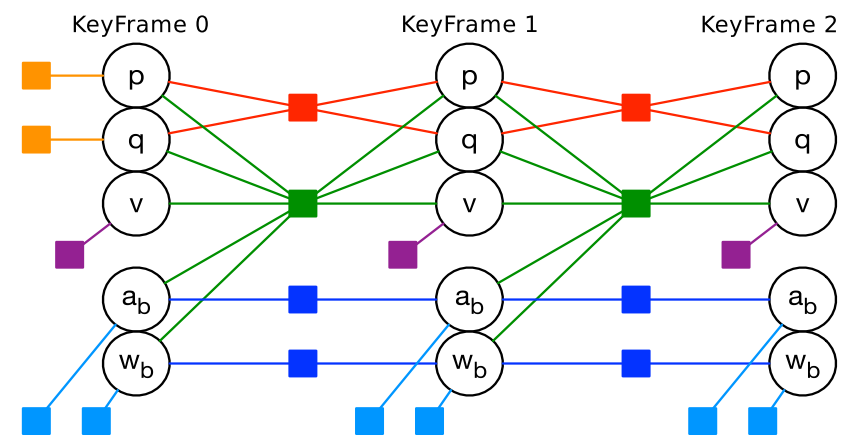

Fig. 1. Detailed factor graph for the initial keyframe and two steps Circles: state blocks for position $(\mathbf{p})$, orientation quaternion $(\mathbf{q})$, velocity $(\mathbf{v})$, accelerometer bias $\left(\mathbf{a}_{b}\right)$, gyrometer bias $\left(\boldsymbol{\omega}_{b}\right)$. Orange: initial pose factor. Red: kinematic factor (deduced from additional sensors). Purple: zero-velocity factor. Green: IMU's delta pre-integration factor. Blue: bias drift factor. Cyan: bias absolute factor.

subtraction operators on the manifold (see e.g. Eq. (25) in Section III, or Eq. (28-31) in Appendix I).

The maximum a posteriori estimation is obtained by iteratively minimizing the Mahalanobis squared norm of all linearized errors

$$
\Delta \mathbf{x}^{*}=\underset{\Delta \mathbf{x}}{\arg \min } \sum_{k}\left\|\mathbf{r}_{k}(\breve{\mathbf{x}})+\mathbf{J}_{k} \Delta \mathbf{x}\right\|_{\mathbf{\Omega}_{k}^{-1}}^{2}
$$

being $\breve{\mathbf{x}}$ the state estimate at the current iteration, and $\mathbf{J}_{k}$ the Jacobian of the $k$-th residual $\mathbf{r}_{k}(\mathbf{x})$ (with $\mathbf{J}_{k}=\partial\left(h_{k}(\mathbf{x}) \ominus \mathbf{z}_{k}\right) / \partial \Delta \mathbf{x}$ in the case of variables lying on a manifold) and $\Omega_{k}$ is the information matrix of the $k$-th measurement. Current methods use the Cholesky [7], [8] or the $\mathrm{QR}$ [9], [10] matrix factorizations to solve for $\Delta \mathrm{x}^{*}$, which is then used to update the current state estimate. The process is iterated until convergence. Incremental methods [8], [10] update the problem directly on the factorized matrices, obtaining important speed-ups.

\section{Keyframe variables}

During a biped walk, we take profit of certain situations where precise and reliable assumptions can be made. For example, the foot velocity is null during its support phase. At these selected instants, we create the keyframes that will produce a chain of states. These states are linked by the measurements, forming our factor graph (Fig. 1). Each keyframe $\mathbf{f}_{i}$ contains the following state blocks: the foot's position, velocity and orientation data, plus the IMU's accelerometer and gyrometer biases,

$$
\mathbf{f}_{i}=\left[\begin{array}{lllll}
\mathbf{p}_{i} & \mathbf{v}_{i} & \mathbf{q}_{i} & \mathbf{a}_{b, i} & \boldsymbol{\omega}_{b, i}
\end{array}\right]^{\top}
$$

\section{Description of factors}

The types of factor considered in our graph are illustrated in Fig. 1. Each factor $k$ requires its own information matrix $\Omega_{k}$, and its residual function $\mathbf{r}_{k}(\mathbf{x})$. These residual functions are detailed hereafter. 
1) Absolute factors: These include initial position and orientation (orange in the figure), zero velocity (purple), and bias magnitude (cyan). Each residual depends on a single state block, which is compared against a reference $\mathbf{z}_{k}$,

$$
\mathbf{r}_{k}\left(\phi_{i}\right)=\phi_{i}-\mathbf{z}_{k}
$$

where $\phi_{i}$ is one among $\left\{\mathbf{p}_{i}, \mathbf{v}_{i}, \mathbf{a}_{b, i}, \boldsymbol{\omega}_{b, i}\right\}$. For the quaternion we implement the residual using the operator $\ominus$ on the sphere of dimension 3 manifold, denoted $S^{3}$ (see (30) in Appendix I for further details),

$$
\mathbf{r}_{k}\left(\mathbf{q}_{i}\right)=\mathbf{q}_{i} \ominus \mathbf{z}_{k}=\log \left(\mathbf{z}_{k}^{*} \otimes \mathbf{q}_{i}\right)
$$

2) Bias drift factors (blue): These are relative factors that allow the bias estimates to drift with time at a controlled rate. Each bias drift residual depends on two state blocks, namely

$$
\begin{aligned}
\mathbf{r}\left(\mathbf{a}_{b, i}, \mathbf{a}_{b, j}\right) & =\mathbf{a}_{b, j}-\mathbf{a}_{b, i} \\
\mathbf{r}\left(\boldsymbol{\omega}_{b, i}, \boldsymbol{\omega}_{b, j}\right) & =\boldsymbol{\omega}_{b, j}-\boldsymbol{\omega}_{b, i}
\end{aligned}
$$

3) Complementary factors (red): These relate position and orientation between two consecutive steps as it can be provided by other sensors than IMU or methods using human walking specificities,

$$
\mathbf{r}\left(\mathbf{p}_{i}, \mathbf{q}_{i}, \mathbf{p}_{j}, \mathbf{q}_{j}\right)=\left[\begin{array}{c}
\mathbf{q}_{i}^{*} \odot\left(\mathbf{p}_{j}-\mathbf{p}_{i}\right)-\mathbf{y}_{k} \\
\log \left(\mathbf{z}_{k}^{*} \otimes \mathbf{q}_{i}^{*} \otimes \mathbf{q}_{j}\right)
\end{array}\right]
$$

where $\mathbf{y}_{k}$ and $\mathbf{z}_{k}$ are respectively the relative position and quaternion measurements.

4) IMU pre-integrated factors (green): These factors are by far the most complex ones and are described in details in the next section.

\section{IMU PRE-INTEGRATION IN $S^{3}$ AND SO(3)}

\section{A. Overview}

Due to the different rates of IMU data and keyframe creations, hundreds of IMU measurements need to be integrated to generate a motion factor linking two consecutive keyframes. In addition to that, the integration of the motion equations in an absolute reference frame strongly depends on the initial conditions of orientation, velocity and IMU bias. Therefore, the changes in the estimates of these magnitudes (inherent to the iterative nature of the optimization) affect the whole motion integral. Delta pre-integration theory was developed to avoid the need of re-integrating all IMU data at each iteration [4], [5]. On the one hand, this theory defines new motion magnitudes called deltas, which are independent of the initial conditions for orientation and velocity, and thus depend only on the IMU data and bias. On the other hand, the effect of the changes in the bias estimates is linearized so that the deltas can be corrected a posteriori, i.e., when computing the residual, using pre-computed Jacobians.

In this section, we revise the IMU pre-integration theory, providing three contributions: 1) a segmentation of the computation pipeline (from measurements, to body magnitudes, to the current delta, and to the integrated delta); 2) a physical interpretation of the delta magnitudes; and 3) a simpler yet rigorous algebraic approach, valid for both the $S^{3}$ (quaternion) and $\mathrm{SO}(3)$ (rotation matrix) manifolds, which takes profit of the pipeline segmentation and the chain rule to compute the otherwise cumbersome Jacobians [5]. Important complements are provided in Appendix I for the sake of background and completeness.

\section{B. State integration in the absolute reference frame}

We define the world-referenced states of the IMU by $\mathbf{x}=(\mathbf{p}, \mathbf{v}, \mathbf{q})$ where $\mathbf{p}$ stands for the position, $\mathbf{v}$ for the velocity and $\mathbf{q}$ for the orientation encoded as a quaternion. The time evolution of $\mathbf{x}$ is governed by the kinematic equation,

$$
\begin{aligned}
& \dot{\mathbf{p}}=\mathbf{v} \\
& \dot{\mathbf{v}}=\mathbf{g}+\mathbf{q} \odot \mathbf{a} \\
& \dot{\mathbf{q}}=\frac{1}{2} \mathbf{q} \otimes \boldsymbol{\omega}
\end{aligned}
$$

where $\mathbf{g}$ denotes the gravity vector and we identify $\mathbf{b}=$ $(\mathbf{a}, \boldsymbol{\omega})$ as the body magnitudes, that is, the magnitudes of acceleration and angular velocity measured by the IMU and expressed in its reference frame. These body magnitudes are obtained at discrete times $t_{j}$ from biased and noisy IMU measurements, i.e.,

$$
\begin{aligned}
& \mathbf{a}_{j} \triangleq \mathbf{a}_{m, j}-\mathbf{a}_{b, j}-\mathbf{a}_{n} \\
& \boldsymbol{\omega}_{j} \triangleq \boldsymbol{\omega}_{m, j}-\boldsymbol{\omega}_{b, j}-\boldsymbol{\omega}_{n},
\end{aligned}
$$

with $\bullet_{m}$ the measurements, $\bullet_{b}$ the biases, and $\bullet_{n}$ the noises. Assuming constant body magnitudes within the IMU sampling period $\delta t \triangleq t_{k}-t_{j}$, we have the discrete-time relation:

$$
\begin{aligned}
& \mathbf{p}_{k}=\mathbf{p}_{j}+\mathbf{v}_{j} \delta t+\frac{1}{2} \mathbf{g} \delta t^{2}+\frac{1}{2} \mathbf{q}_{j} \odot \mathbf{a}_{j} \delta t^{2} \\
& \mathbf{v}_{k}=\mathbf{v}_{j}+\mathbf{g} \delta t+\mathbf{q}_{j} \odot \mathbf{a}_{j} \delta t \\
& \mathbf{q}_{k}=\mathbf{q}_{j} \otimes \operatorname{Exp}\left(\boldsymbol{\omega}_{j} \delta t / 2\right)
\end{aligned}
$$

\section{Delta definitions}

Consider a non-rotating reference frame that is free-falling at the acceleration of gravity $\mathbf{g}$, and name it $\mathcal{G}_{t}$. An ideal (unbiased and noiseless) IMU glued to this frame would measure null linear accelerations and angular velocities. Any non-null measurements would be due to a relative motion of the IMU with respect to $\mathcal{G}_{t}$.

At a given keyframe instant $t_{i}$, we initialize $\mathcal{G}_{i}$ at $\mathbf{x}_{i}=\left(\mathbf{p}_{i}, \mathbf{v}_{i}, \mathbf{q}_{i}\right)$. At a later keyframe instant $t_{j}(j>i), \mathcal{G}_{j}$ has fallen according to $\mathbf{g}$, and the state of our moving body is now at $\mathbf{x}_{j}=\left(\mathbf{p}_{j}, \mathbf{v}_{j}, \mathbf{q}_{j}\right)$. The motion variation, denoted $\Delta_{i j}$, is defined as the state variation in position, velocity and orientation of our body between $\mathcal{G}_{i}$ and $\mathcal{G}_{j}$, that is,

$$
\begin{aligned}
\Delta \mathbf{p}_{i j} & =\mathbf{q}_{i}^{*} \odot\left(\mathbf{p}_{j}-\mathbf{p}_{i}-\mathbf{v}_{i} \Delta t_{i j}-\frac{1}{2} \mathbf{g} \Delta t_{i j}^{2}\right) \\
\Delta \mathbf{v}_{i j} & =\mathbf{q}_{i}^{*} \odot\left(\mathbf{v}_{j}-\mathbf{v}_{i}-\mathbf{g} \Delta t_{i j}\right) \\
\Delta \mathbf{q}_{i j} & =\mathbf{q}_{i}^{*} \otimes \mathbf{q}_{j}
\end{aligned}
$$

where $\Delta t_{i j} \triangleq t_{j}-t_{i}$ is the time duration between the two keyframes. Notice that this definition of $\Delta_{i j}$ is the same as provided in [4], [5], and we have given it here a clear physical meaning. It is worth to notice that the deltas form a 
group under the composition law $\Delta_{i k} \triangleq \Delta_{i j} \oplus \Delta_{j k}$, defined by:

$$
\begin{aligned}
& \Delta \mathbf{p}_{i k}=\Delta \mathbf{p}_{i j}+\Delta \mathbf{v}_{i j} \Delta t_{j k}+\Delta \mathbf{q}_{i j} \odot \Delta \mathbf{p}_{j k} \\
& \Delta \mathbf{v}_{i k}=\Delta \mathbf{v}_{i j}+\Delta \mathbf{q}_{i j} \odot \Delta \mathbf{v}_{j k} \\
& \Delta \mathbf{q}_{i k}=\Delta \mathbf{q}_{i j} \otimes \Delta \mathbf{q}_{j k}
\end{aligned}
$$

with identity $\Delta_{0}=[(0,0,0),(0,0,0),(1,0,0,0)]$, and inverse $\Delta_{j i} \triangleq \Delta_{i j}^{-1}$ shuch that $\Delta^{-1} \oplus \Delta=\Delta \oplus \Delta^{-1}=\Delta_{0}$ (the inverse expression is not given for space reasons). At any time $j$ we can recover the state estimate $\mathbf{x}_{j}$ from the state estimate $\mathbf{x}_{i}$ and the motion delta $\Delta_{i j}$ :

$$
\begin{aligned}
& \mathbf{p}_{j}=\mathbf{p}_{i}+\mathbf{v}_{i} \Delta t_{i j}+\frac{1}{2} \mathbf{g} \Delta t_{i j}^{2}+\mathbf{q}_{i} \odot \Delta \mathbf{p}_{i j} \\
& \mathbf{v}_{j}=\mathbf{v}_{i}+\mathbf{g} \Delta t_{i j}+\mathbf{q}_{i} \odot \Delta \mathbf{v}_{i j} \\
& \mathbf{q}_{j}=\mathbf{q}_{i} \otimes \Delta \mathbf{q}_{i j}
\end{aligned}
$$

\section{Incremental delta pre-integration}

Substituting the integration Eq. (11) in the delta definitions (12), we obtain the incremental delta pre-integration,

$$
\begin{aligned}
\Delta \mathbf{p}_{i k} & =\Delta \mathbf{p}_{i j}+\Delta \mathbf{v}_{i j} \delta t+\frac{1}{2} \Delta \mathbf{q}_{i j} \odot \mathbf{a}_{j} \delta t^{2} \\
\Delta \mathbf{v}_{i k} & =\Delta \mathbf{v}_{i j}+\Delta \mathbf{q}_{i j} \odot \mathbf{a}_{j} \delta t \\
\Delta \mathbf{q}_{i k} & =\Delta \mathbf{q}_{i j} \otimes \operatorname{Exp}\left(\boldsymbol{\omega}_{j} \delta t\right)
\end{aligned}
$$

with $\Delta_{i i}=\Delta_{0}$. Interestingly, (15) is analogous to the motion of a body in an inertial frame under constant acceleration and rotation rate. Notice that by letting the reference frame fall with gravity, we get rid of the dependence on gravity in the integration equations, and only the body magnitudes drive the integral. Indeed, we can define a proper delta $\delta_{j k}$ from the current body magnitudes $\mathbf{b}_{j}=\left(\mathbf{a}_{j}, \boldsymbol{\omega}_{j}\right) \triangleq \mathbf{b}_{m, i}-\mathbf{b}_{b, j}-\mathbf{b}_{n, j}$ at time $t_{j}$,

$$
\begin{aligned}
\delta \mathbf{p}_{j k} & =\frac{1}{2} \mathbf{a}_{j} \delta t^{2} \\
\delta \mathbf{v}_{j k} & =\mathbf{a}_{j} \delta t \\
\delta \mathbf{q}_{j k} & =\operatorname{Exp}\left(\boldsymbol{\omega}_{j} \delta t\right)
\end{aligned}
$$

and write the integration (15) as the composition

$$
\Delta_{i k}=\Delta_{i j} \oplus \delta_{j k}
$$

described in (13). Typically, we take the biases at the keyframe time $t_{i}$, that is, $\mathbf{b}_{b, j}=\mathbf{b}_{b, i}$. In the following, we will identify $\Delta$ with the pre-integrated delta, and $\delta$ with the current delta.

\section{E. Jacobians}

We note all Jacobians with $\mathbf{J}_{x}^{y} \triangleq \partial y / \partial x$ and refer the reader to Appendix I for details on the development of all non-trivial Jacobian blocks in this section.

1) Jacobians of the body magnitudes: from Eq. (10) we have:

$$
\mathbf{J}_{\mathbf{b}_{m}}^{\mathbf{b}}=\mathbf{I}_{6} \quad \mathbf{J}_{\mathbf{b}_{b}}^{\mathbf{b}}=-\mathbf{I}_{6} \quad \mathbf{J}_{\mathbf{b}_{n}}^{\mathbf{b}}=-\mathbf{I}_{6} .
$$

2) Jacobians of the current delta: We have from (16),

$$
\mathbf{J}_{\mathbf{b}_{j}}^{\delta_{j k}}=\left[\begin{array}{cc}
\frac{1}{2} \mathbf{I} \delta t^{2} & \mathbf{0} \\
\mathbf{I} \delta t & \mathbf{0} \\
\mathbf{0} & \mathbf{J}_{r}\left(\boldsymbol{\omega}_{j} \delta t\right) \delta t
\end{array}\right] \in \mathbb{R}^{9 \times 6}
$$

where we develop the lower-right block as in Appendix IE.1.

3) Jacobians of the delta composition: We differentiate the delta composition (17) described in (13),

$$
\begin{aligned}
& \mathbf{J}_{\Delta_{i j}}^{\Delta_{i k}}=\left[\begin{array}{ccc}
\mathbf{I} & \mathbf{I} \delta t & -\Delta \mathbf{R}_{i j}\left[\delta \mathbf{p}_{j k}\right]_{\times} \\
\mathbf{0} & \mathbf{I} & -\Delta \mathbf{R}_{i j}\left[\delta \mathbf{v}_{j k}\right]_{\times} \\
\mathbf{0} & \mathbf{0} & \delta \mathbf{R}_{j k}^{\top}
\end{array}\right] \in \mathbb{R}^{9 \times 9} \\
& \mathbf{J}_{\delta_{j k}}^{\Delta_{i k}}=\left[\begin{array}{ccc}
\Delta \mathbf{R}_{i j} & \mathbf{0} & \mathbf{0} \\
\mathbf{0} & \Delta \mathbf{R}_{i j} & \mathbf{0} \\
\mathbf{0} & \mathbf{0} & \mathbf{I}
\end{array}\right] \quad \in \mathbb{R}^{9 \times 9}
\end{aligned}
$$

where $\Delta \mathbf{R}_{i j}$ and $\delta \mathbf{R}_{j k}$ are the rotation matrix deltas corresponding to the respective quaternion deltas $\Delta \mathbf{q}_{i j}$ and $\delta \mathbf{q}_{j k}$. We develop all the non-trivial blocks as in Appendix I-E.2 and Appendix I-E.3.

\section{F. Incremental delta covariance integration}

Let $\mathbf{Q}_{\Delta}$ be the covariance of the pre-integrated delta, and $\mathbf{Q}_{n}$ the one of the measurement noise. For convenience, we first compute the covariance of the current delta,

$$
\mathbf{Q}_{\delta}=\mathbf{J}_{\mathbf{b}_{n}}^{\delta} \mathbf{Q}_{n} \mathbf{J}_{\mathbf{b}_{n}}^{\delta}{ }^{\top},
$$

where $\mathbf{J}_{\mathbf{b}_{n}}^{\delta}=\mathbf{J}_{\mathbf{b}}^{\delta} \cdot \mathbf{J}_{\mathbf{b}_{n}}^{\mathbf{b}}$ is the noise Jacobian, obtained with (18-19) and the chain rule. The delta covariance is then integrated with

$$
\mathbf{Q}_{\Delta_{i k}}=\mathbf{J}_{\Delta_{i j}}^{\Delta_{i k}} \mathbf{Q}_{\Delta_{i j}} \mathbf{J}_{\Delta_{i j}}^{\Delta_{i k}^{\top}}+\mathbf{J}_{\delta_{j k}}^{\Delta_{i k}} \mathbf{Q}_{\delta} \mathbf{J}_{\delta_{j k}}^{\Delta_{i k}}{ }^{\top}
$$

using Jacobians (20), and starting at $\mathbf{Q}_{\Delta_{i i}}=\mathbf{0}_{9 \times 9}$.

\section{G. Delta correction with new bias}

Let $\bar{\Delta}$ and $\overline{\mathbf{b}_{b}}$ be respectively the pre-integrated delta and the bias values used during pre-integration. Since the bias estimates change at each iteration of the optimizer, we need to update the delta according to the new bias values $\mathbf{b}_{b}$. We do so with the linearized update,

$$
\Delta=\bar{\Delta}+\mathbf{J}_{\mathbf{b}_{b}}^{\Delta}\left(\mathbf{b}_{b}-\overline{\mathbf{b}_{b}}\right),
$$

where $\mathbf{J}_{\mathbf{b}_{b}}^{\Delta}$ is the pre-integrated bias Jacobian, computed incrementally using also the chain rule,

$$
\mathbf{J}_{\mathbf{b}_{b}}^{\Delta_{i k}}=\mathbf{J}_{\Delta_{i j}}^{\Delta_{i k}} \mathbf{J}_{\mathbf{b}_{b}}^{\Delta_{i j}}-\mathbf{J}_{\delta_{j k}}^{\Delta_{i k}} \mathbf{J}_{\mathbf{b}_{b}}^{\delta_{j k}} .
$$

with $\mathbf{J}_{\mathbf{b}_{b}}^{\delta_{j k}}=\mathbf{J}_{\mathbf{b}}^{\delta_{j k}} \mathbf{J}_{\mathbf{b}_{b}}^{\mathbf{b}}$. This Jacobian starts at $\mathbf{J}_{\mathbf{b}_{b}}^{\Delta_{i i}}=\mathbf{0}_{9 \times 9}$.

\section{H. Residuals}

The computation of the residuals for the IMU delta factors (see Fig. 1, green) requires: the state estimates $\mathbf{x}_{i}$ and $\mathbf{x}_{j}$; the current bias estimates $\mathbf{b}_{b, i}$; the pre-integrated delta $\overline{\Delta_{i j}}$; the bias used during pre-integration $\overline{\mathbf{b}_{b, i}}$; and the pre-integrated bias Jacobian $\mathbf{J}_{\mathbf{b}_{b}}^{\Delta_{i j}}$. The process is best understood if split into smaller steps: we first compute a corrected delta $\Delta_{i j}$ 
using (23); then we compute a predicted delta $\widehat{\Delta}_{i j}$ using (12); and finally we compute the residual with

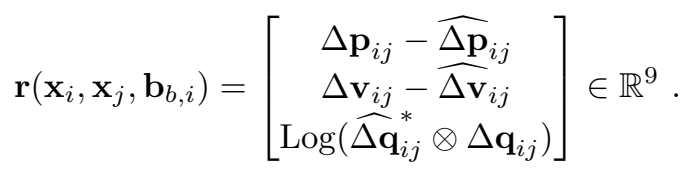

Its information matrix is given by $\boldsymbol{\Omega}=\mathbf{Q}_{\Delta i k}^{-1}$.

\section{EXPERIMENTS}

We use the dataset made available thanks to Angermann et. al. [11] to validate our method and compare the results to provided outputs from the state-of-the-art Kalman filter. Our method is applied on several scenarios from these dataset and we present typical results obtained with two representative cases. The first sets of data correspond to a human walking back and forth while the second example is related to a walking pattern describing a eight-shape. Both IMU and motion capture data are provided at $100 \mathrm{~Hz}$.

Then, we will investigate the use of additional sensors to demonstrate the feasibility of fusion strategies using a low cost IMU running at $1 \mathrm{kHz}$ (Invensense's MPU6050 [12]).

\section{A. Method}

Keyframes are created at the beginning and ending of each support phase of the selected foot according to ZUPT detection provided by the dataset. Factors active in the graph (see Fig. 1) are: initial position and yaw; zero velocity, bias drift, and IMU pre-integration. We only use the bias magnitude and zero velocity constraint factors in the initial keyframe. All the graph is optimized after each keyframe creation so that these estimates can be used for future estimations with new keyframes. Zero velocity constraints are applied as a fixed prior for the optimizer, meaning that corresponding variables will not be estimated but considered as parameters. As a consequence, we need to be confident that timestamps detected as zero velocity instants really match static phases of the IMU. Otherwise we would be imposing a constraint inducing a wrong estimate of the trajectory.

In order to show how interesting fusion strategies can be, we simulate the use of an odometry sensor providing the displacement of the foot between two frames at a frequency that is lower than IMU. This specific case is a common use of IMUs in fusion strategies to reduce the drift error due to IMU's biases [13]. With a better estimation of its biases, the IMU can then be used to detect events related the human wearing the device. Such strategy can be implemented with RFID sensors or more basically with visual odometry. In our case, we use motion capture information to reconstruct odometry between consecutive keyframes, allowing us to create keyframes during the flying phases of the foot. As in the usual ZUPT-aided inertial navigation, zero-velocity constraints are imposed only on keyframes created during contact phases. Kinematic odometry is also added between all consecutive keyframes (see Fig. 1).

\section{B. Results}

1) State estimation using ZUPT only: As motion capture data are not used during the experiments, the system is not provided with enough information to be able to precisely estimate the vertical orientation of the IMU due to its nonobservability from the IMU measurements directly. As a consequence, the estimation of the state of the foot is not able to converge to its real value. In other words, the foot trajectory can be recovered up to a rotation around the gravity axis. In the following, we manually translate the first estimated states to the initial reference one and rotate the estimated trajectories in order to compare our results against the ground-truth measurements. Our system is able to estimate the state of the system to a precision close to the state-of-the-art 9-state Kalman filter [14] as shown in Fig. 3.

Besides, the experiment shows satisfying results in terms of computational times. Indeed, 3.1 seconds are enough to integrate all the data corresponding to a 50 seconds experiment, that is more than 5000 IMU data, to build and to optimize the graph each time a keyframe is created with our framework, with a total of 80 keyframes. It would be hard, if not currently impossible, to get such results using the IMU without a proper pre-integration method. The optimization part is currently handled by Google Ceres optimizer [6] using the sparse structure of the problem. All in all, it takes $98 \mu \mathrm{s}$ in average to read and integrate a single IMU measurement including the computation of jacobians.

2) State estimation using ZUPT and sensor fusion: The strength of the optimal estimation can also be found in the fusion strategies. Fig. 2 shows the reconstructed foot trajectories for the two cases: without and with the flying keyframe information. Adding kinematic information between keyframes enhances the observability of the system and allows to a better estimation. A major advantage of IMU's pre-integration theory is the ability to use past preintegrations corrected with current estimates of keyframes and biases as simply as it takes to integrate a single IMU data. This removes the need of linearization of intermediate IMU data between previous and current estimates of the state on which the integration must be performed. We also notice that the use of a flying keyframe makes the bias estimation more stable.

\section{RELATED WORK}

Person localization using a foot-mounted IMU was first introduced in [15]. Pedestrian Dead Reckoning (PDR) methods (aka Personal Navigation Devices), make use of one or more IMU installed on the body of the subject. The main idea of PDR techniques is to integrate inertial measurements with Zero Velocity Update (ZUPT) constraints to reduce errors [16]. This work is extensively used in IMU-based human localization works and various fields [17] analyzes the gait of a walking person with PDR method to estimate the direction of the shoe, thus the walking direction, and measure stride length. Shoe-mounted IMU is still considered as a possible way to accurately localize persons in an indoor environment 

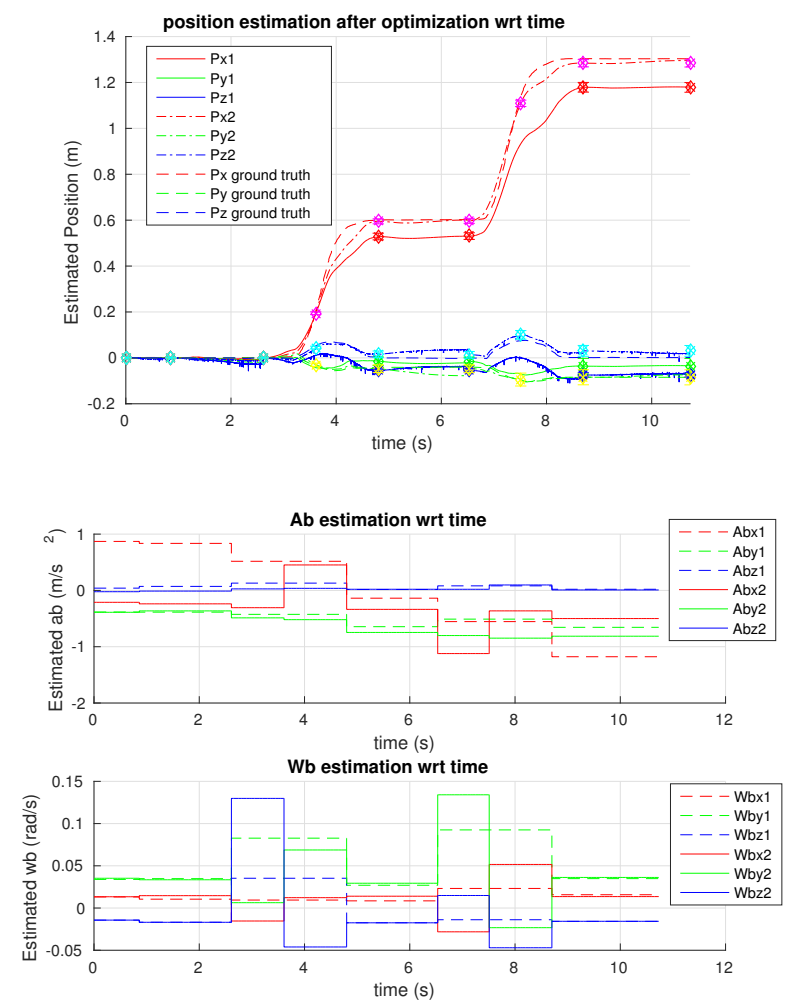

Fig. 2. Top: trajectory estimation during human walking with an IMU attached to a foot. Continuous, dashed and dashed-dot lines are respectively: ground truth, estimation with zero velocity constraints only, estimation using zero velocity constraint and 1 odometry measurement during foot's flying phase. Odometry was here built from motion capture data. Bottom: Evolution of corresponding estimated IMU biases

due to lower drift errors when compared to body-mounted solutions [18].

Various strategies can be considered to improve the localization results of foot-mounted IMU navigation. Prior information can be exploited when merging the measurements of several IMUs, for example relative to the maximum step length the pedestrian could do when using two foot-mounted IMUs [19].

Fusion strategies with information coming from different sensors can also be used to improve localization results as it is already done in robotics: GPS information [20], [21], [22], received signal strength indicator (RSSI) from wireless communication [23] using wireless local area networks or radio-frequency identification (RFID) tags placed at known locations [24] along with other drift reduction methods such as zero velocity updates, zero angular-rate updates (ZARU) and the use of magnetometers. Using these strategies might be a successful solution to overcome the drift observed in methods using IMU and to obtain positioning errors of approximately $1.5 \mathrm{~m}$ [24]. In [25], a foot-mounted IMU is fused with a waist-mounted visual odometry system to update the state of the system composed of its position, velocity and acceleration. This last system was used recently [26] to design a fusion strategy requiring measurements only once per human step instead of every time step.
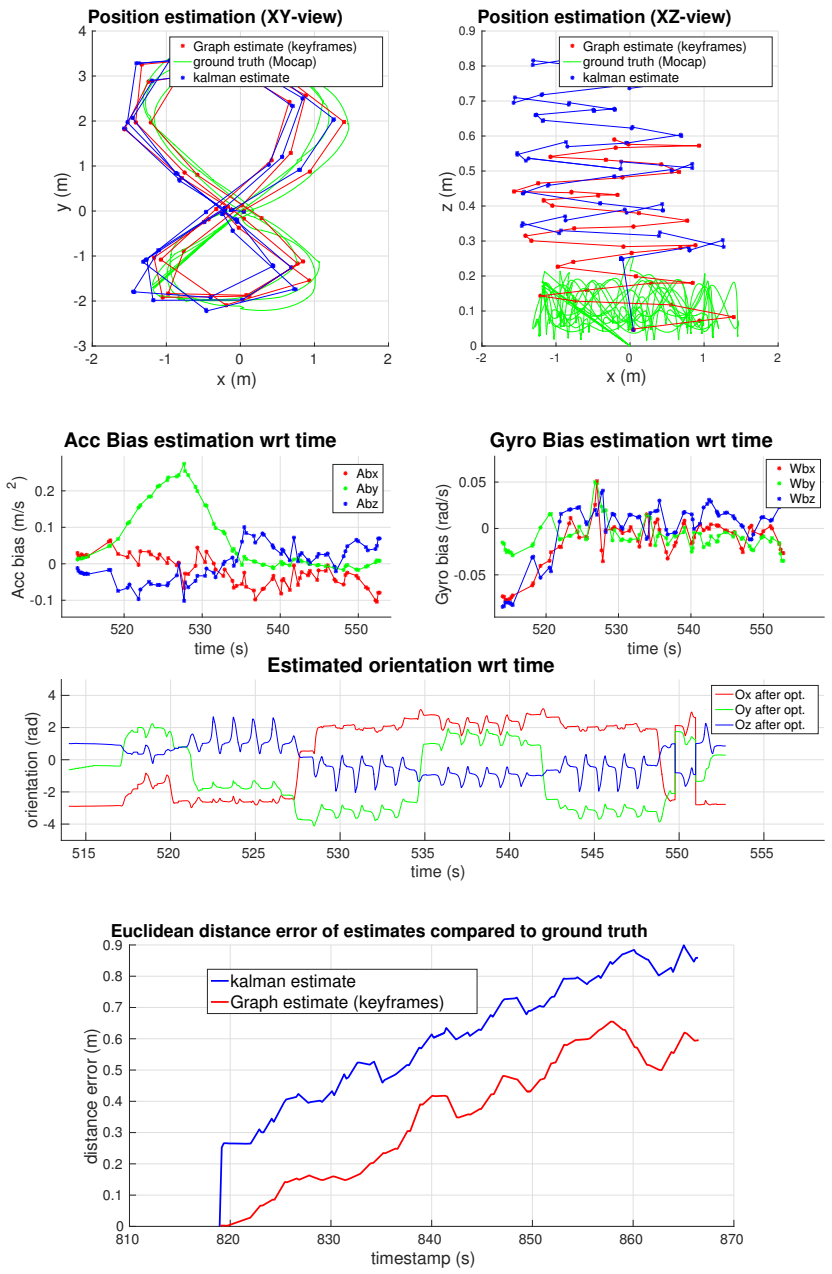

Fig. 3. Top: trajectory estimation during human 8-shaped walking phases with an IMU attached to a foot. Stars in blue and red colors are respectively the reference estimated states provided in the dataset and the estimation with our approach. The continuous green line is the ground truth as given by the motion capture system. Middle: Evolution of estimated IMU biases and orientation of the device. Biases are estimated only for keyframes and considered as constant between them. Bottom: Evolution of euclidean distance errors between estimates and the ground truth for this 8-shaped walking trajectory.

More information can be structured into a map following a SLAM approach [27], leading to a bounded error growth to 1 meter. This FootSLAM uses dynamic Bayesian network and loop-closure strategies. The idea exploited here is to use the normal human behavior consisting on relying on visual information to guide the motion and avoid obstacles.

Previously cited example tend to show how important it is for pedestrian inertial navigation system to be able to deal with the localization drifts due to the integration of IMU's data. From this analysis, we see that two important aspects have been investigated to solve pedestrian localization: i) exploiting some specificities of human behavior with the inertial measurements as prior knowledge and ii) fusing IMU with additional complementary sensors. In this paper, we have shown that using a graphical model is a sane and efficient way to encode prior knowledge about the human behavior (horizontal foot during zero-velocity phases). While 
we are not exploiting any absolute measurement, the drift resulting of the odometry integration is contained to some reasonable margin (i.e. comparable to the accuracy obtained when a rough map is used). An additional feature of our approach is that it is easy to extend the graphical model, either with additional prior knowledge, or with measurements coming from additional sensors. For example, fusing absolute but noisy measurements like GPS, RFID or RSSI would be straight forward.

\section{Conclusion}

We have presented a method to measure foot movement during an IMU attached to the foot and exploit available knowledge extracted from the gait phases, such as zero velocity and IMU bias dynamics. Measurements and prior knowledge have been described in a graphical model where the full IMU state (position, orientation, velocity, bias) is observable. We then used nonlinear optimization techniques based on factor graphs, which has proved to be a flexible and powerful fusion framework. For this, we have revised the IMU pre-integration theory, and proposed an implementation in the quaternions manifold, with simpler derivations than previous works, and with physical interpretations, which we believe go in the direction of improving the clarity of the method. Results showed that this estimation method is able to properly estimate the bias, then leading to an accurate odometry where the drift remains reasonable, even after minutes of integration. The method easily extends to additional prior knowledge or additional sensors. We also plan to use it to accurately track the odometry of a biped robot, while fusing the inertial measurements of the robot feet with the encoders measurements of its kinematic chain. Further work are needed to make the system less critical to wrong ZUPT detections. To achieve this goal we can change the ZUPT implementation strategy from a fixed parameter to an estimated one starting with a zero prior and low variance.

\section{APPENDIX I}

\section{DEFINITION OF THE DERIVATIVES IN $S^{3}$ AND $S O(3)$}

\section{A. Exp and Log maps in $S^{3}$ and $S O(3)$}

We use vectorized versions of the exponential and logarithmic maps in the rotation groups $S^{3}$ (quaternion) and $S O(3)$ (rotation matrix), and denote them with capitalized names $\operatorname{Exp}()$ and $\log ()$ (see Fig. 4, left). They operate directly on the vector space $\mathbb{R}^{3}$, and use either quaternions for $S^{3}$,

$$
\begin{gathered}
\mathbf{q}=\operatorname{Exp}(\boldsymbol{\theta}) \triangleq\left[\begin{array}{c}
\cos (\theta / 2) \\
\mathbf{u} \sin (\theta / 2)
\end{array}\right] \\
\theta \mathbf{u}=\log (\mathbf{q}) \triangleq 2 \mathbf{q}_{v} \frac{\arctan \left(\left\|\mathbf{q}_{v}\right\|, q_{w}\right)}{\left\|\mathbf{q}_{v}\right\|},
\end{gathered}
$$

where $\mathbf{q} \triangleq\left(q_{w}, \mathbf{q}_{v}\right)$, or rotation matrices for $S O(3)$,

$$
\begin{aligned}
\mathbf{R} & =\operatorname{Exp}(\boldsymbol{\theta}) \triangleq \mathbf{I}+\sin \theta[\mathbf{u}]_{\times}+(1-\cos \theta)[\mathbf{u}]_{\times}^{2} \\
\theta \mathbf{u} & =\log (\mathbf{R}) \triangleq \frac{\theta\left(\mathbf{R}-\mathbf{R}^{\top}\right)^{\vee}}{2 \sin \theta},
\end{aligned}
$$

with $\theta=\cos ^{-1}\left(\frac{\operatorname{trace}(\mathbf{R})-1}{2}\right)$, and where $\bullet \vee$, known as the vee operator, is the inverse of the skew operator $[\bullet]_{X}$. Their exact form ( $\mathbf{q}$ or $\mathbf{R}$ ) is always clear by the context. Since the quaternion
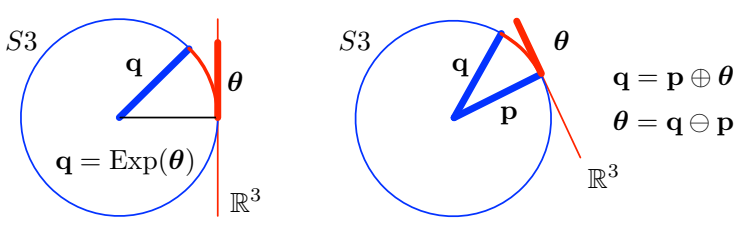

Fig. 4. The $S^{3}$ manifold is a unit sphere in $\mathbb{R}^{4}$, here represented by a unit circle (blue), where all unit quaternions live. The tangent space to the manifold is the hyperplane $\mathbb{R}^{3}$, here represented by a line (red). The $\operatorname{Exp}()$ and $\log ()$ operators map elements of $\mathbb{R}^{3}$ to/from elements of $S^{3}$. The $\oplus$ and $\ominus$ operators relate elements of the manifold with elements in the tangent space. (Likewise, these figures illustrate the $S O(3)$ manifold.)

implementation is one of our contributions, in the following we will refer to the rotation groups $S^{3}$ and $S O(3)$ with the unique name $S^{3}$, although everything applies equally to $S O(3)$.

\section{B. The additive and subtractive operators in $S^{3}$ and $S O(3)$}

The 'plus' operator, $\oplus: S^{3} \times \mathbb{R}^{3} \rightarrow S^{3}$, composes a reference element $\mathrm{R} \in S^{3}$ with a (often small) rotation specified by a vector of $\boldsymbol{\theta} \in \mathbb{R}^{3}$ that is tangent to the $S^{3}$ manifold at $\mathrm{R}$, yielding an element $\mathrm{S} \in S^{3}$ (see Fig. 4, right). The 'minus' operator, $\ominus$ : $S^{3} \times S^{3} \rightarrow \mathbb{R}^{3}$ is the inverse of the above. These operators are defined for both $\mathbf{q}$ and $\mathbf{R}$,

$$
\begin{aligned}
& \mathbf{q}=\mathbf{p} \oplus \boldsymbol{\theta} \triangleq \mathbf{p} \otimes \operatorname{Exp}(\boldsymbol{\theta}) \\
& \mathbf{S}=\mathbf{R} \oplus \boldsymbol{\theta} \triangleq \mathbf{R} \operatorname{Exp}(\boldsymbol{\theta}) \\
& \boldsymbol{\theta}=\mathbf{q} \ominus \mathbf{p} \triangleq \log \left(\mathbf{p}^{*} \otimes \mathbf{q}\right) \\
& \boldsymbol{\theta}=\mathbf{S} \ominus \mathbf{R} \triangleq \log \left(\mathbf{R}^{\top} \mathbf{S}\right) .
\end{aligned}
$$

\section{The four possible derivative definitions}

For functions $f: \mathbb{R}^{m} \rightarrow \mathbb{R}^{n}$, the derivative is defined classically using the standard operators $\{+,-\}$,

$$
\frac{\partial f(\mathbf{x})}{\partial \mathbf{x}} \triangleq \lim _{\delta \mathbf{x} \rightarrow 0} \frac{f(\mathbf{x}+\delta \mathbf{x})-f(\mathbf{x})}{\delta \mathbf{x}} \quad \in \mathbb{R}^{n \times m} ;
$$

for functions $g: S^{3} \rightarrow S^{3}$, we use the operators $\{\oplus, \ominus\}$,

$$
\frac{\partial g(\mathrm{R})}{\partial \boldsymbol{\theta}} \triangleq \lim _{\delta \boldsymbol{\theta} \rightarrow 0} \frac{g(\mathrm{R} \oplus \delta \boldsymbol{\theta}) \ominus g(\mathrm{R})}{\delta \boldsymbol{\theta}} \quad \in \mathbb{R}^{3 \times 3}
$$

for functions $h: \mathbb{R}^{m} \rightarrow S^{3}$, we use $\{+, \ominus\}$,

$$
\frac{\partial h(\mathbf{x})}{\partial \mathbf{x}} \triangleq \lim _{\delta \mathbf{x} \rightarrow 0} \frac{h(\mathbf{x}+\delta \mathbf{x}) \ominus h(\mathbf{x})}{\delta \mathbf{x}} \quad \in \mathbb{R}^{3 \times m} ;
$$

and for functions $k: S^{3} \rightarrow \mathbb{R}^{n}$, we use $\{\oplus,-\}$,

$$
\frac{\partial k(\mathrm{R})}{\partial \boldsymbol{\theta}} \triangleq \lim _{\delta \boldsymbol{\theta} \rightarrow 0} \frac{k(\mathrm{R} \oplus \delta \boldsymbol{\theta})-k(\mathrm{R})}{\delta \boldsymbol{\theta}} \quad \in \mathbb{R}^{n \times 3} .
$$

It might be worth noticing that all these Jacobians are independent of the representation chosen $\left(S^{3}\right.$ or $\left.S O(3)\right)$.

\section{Right Jacobian of $S^{3}$ and $S O(3)$}

We define the right Jacobian as,

$$
\mathbf{J}_{r}(\boldsymbol{\theta}) \triangleq \frac{\partial \operatorname{Exp}(\boldsymbol{\theta})}{\partial \boldsymbol{\theta}} \in \mathbb{R}^{3 \times^{3}}
$$

and implement it using (34). It admits the closed form [28, pag. 40],

$$
\mathbf{J}_{r}(\boldsymbol{\theta})=\mathbf{I}-\frac{1-\cos \|\boldsymbol{\theta}\|}{\|\boldsymbol{\theta}\|^{2}}[\boldsymbol{\theta}]_{\times}+\frac{\|\boldsymbol{\theta}\|-\sin \|\boldsymbol{\theta}\|}{\|\boldsymbol{\theta}\|^{3}}[\boldsymbol{\theta}]_{\times}^{2} .
$$




\section{E. Examples}

1) Function $\mathbb{R}^{3} \rightarrow S^{3}$ : The function $f(\boldsymbol{\omega})=\operatorname{Exp}(\boldsymbol{\omega} \delta t)$ produces elements of $S^{3}$ from vectors $\omega \in \mathbb{R}^{3}$. Its Jacobian with respect to $\boldsymbol{\omega}$ follows from (34), but is better obtained from (36) and the chain rule,

$$
\frac{\partial \operatorname{Exp}(\boldsymbol{\omega} \delta t)}{\partial \boldsymbol{\omega}}=\frac{\partial \operatorname{Exp}(\boldsymbol{\omega} \delta t)}{\partial(\boldsymbol{\omega} \delta t)} \frac{\partial(\boldsymbol{\omega} \delta t)}{\partial \boldsymbol{\omega}}=\mathbf{J}_{r}(\boldsymbol{\omega} \delta t) \delta t .
$$

2) Function $S^{3} \times \mathbb{R}^{3} \rightarrow \mathbb{R}^{3}$ : The rotation $f(\mathrm{R}, \mathbf{v})=\mathbf{q} \odot \mathbf{v}=$ $\mathbf{R} \mathbf{v}$ produces vectors of $\mathbb{R}^{3}$ from elements $\mathrm{R} \in S^{3}$ and vectors $\mathbf{v} \in \mathbb{R}^{3}$. The first Jacobian is defined by (35) and developed as

$$
\begin{gathered}
\frac{\partial \mathbf{q} \odot \mathbf{v}}{\partial \boldsymbol{\theta}}=\frac{\partial \mathbf{R} \mathbf{v}}{\partial \boldsymbol{\theta}} \triangleq \lim _{\delta \boldsymbol{\theta} \rightarrow 0} \frac{(\mathbf{R} \oplus \delta \boldsymbol{\theta}) \mathbf{v}-\mathbf{R} \mathbf{v}}{\delta \boldsymbol{\theta}} \\
=\lim _{\delta \boldsymbol{\theta} \rightarrow 0} \frac{\mathbf{R} \operatorname{Exp}(\delta \boldsymbol{\theta}) \mathbf{v}-\mathbf{R} \mathbf{v}}{\delta \boldsymbol{\theta}}=\lim _{\delta \boldsymbol{\theta} \rightarrow 0} \frac{\mathbf{R} \cdot\left(\mathbf{I}+[\delta \boldsymbol{\theta}]_{\times}\right) \mathbf{v}-\mathbf{R} \mathbf{v}}{\delta \boldsymbol{\theta}} \\
=\lim _{\delta \boldsymbol{\theta} \rightarrow 0} \frac{\mathbf{R}[\delta \boldsymbol{\theta}]_{\times} \mathbf{v}}{\delta \boldsymbol{\theta}}=\lim _{\delta \boldsymbol{\theta} \rightarrow 0} \frac{-\mathbf{R}[\mathbf{v}]_{\times} \delta \boldsymbol{\theta}}{\delta \boldsymbol{\theta}}=-\mathbf{R}[\mathbf{v}]_{\times}
\end{gathered}
$$

where we used the properties $\operatorname{Exp}(\delta \boldsymbol{\theta}) \approx \mathbf{I}+[\delta \boldsymbol{\theta}]_{\times}$and $[\mathbf{a}]_{\times} \mathbf{b}=$ $-[\mathbf{b}]_{\times}$a. The second Jacobian is defined by (32) and yields,

$$
\frac{\partial \mathbf{q} \odot \mathbf{v}}{\partial \mathbf{v}}=\frac{\partial \mathbf{R} \mathbf{v}}{\partial \mathbf{v}} \triangleq \lim _{\partial \mathbf{v} \rightarrow 0} \frac{\mathbf{R} \cdot(\mathbf{v}+\partial \mathbf{v})-\mathbf{R} \mathbf{v}}{\partial \mathbf{v}}=\mathbf{R}
$$

3) Function $S^{3} \times S^{3} \rightarrow S^{3}$ : The function $f(\mathrm{Q}, \mathrm{R})=\mathbf{q} \otimes \mathbf{r}=$ $\mathbf{Q} \mathbf{R}$ produces rotation composition. Its Jacobians are computed from (33), using the property $\operatorname{Exp}(\mathbf{R} \boldsymbol{\theta})=\mathbf{R} \operatorname{Exp}(\boldsymbol{\theta}) \mathbf{R}^{\top}$,

$$
\begin{aligned}
\frac{\partial \mathbf{q}(\boldsymbol{\theta}) \otimes \mathbf{r}}{\partial \boldsymbol{\theta}}=\frac{\partial \mathbf{Q}(\boldsymbol{\theta}) \mathbf{R}}{\partial \boldsymbol{\theta}} & =\lim _{\delta \boldsymbol{\theta} \rightarrow 0} \frac{\log \left((\mathbf{Q R})^{\top}(\mathbf{Q} \operatorname{Exp}(\delta \boldsymbol{\theta}) \mathbf{R})\right)}{\delta \boldsymbol{\theta}} \\
& =\lim _{\delta \boldsymbol{\theta} \rightarrow 0} \frac{\log \left(\mathbf{R}^{\top} \operatorname{Exp}(\delta \boldsymbol{\theta}) \mathbf{R}\right)}{\delta \boldsymbol{\theta}} \\
& =\lim _{\delta \boldsymbol{\theta} \rightarrow 0} \frac{\log \left(\operatorname{Exp}\left(\mathbf{R}^{\top} \delta \boldsymbol{\theta}\right)\right)}{\delta \boldsymbol{\theta}}=\mathbf{R}^{\top}, \\
\frac{\partial \mathbf{q} \otimes \mathbf{r}(\boldsymbol{\phi})}{\partial \boldsymbol{\phi}}=\frac{\partial \mathbf{Q} \mathbf{R}(\boldsymbol{\phi})}{\partial \boldsymbol{\phi}} & =\lim _{\delta \boldsymbol{\phi} \rightarrow 0} \frac{\log \left((\mathbf{Q R})^{\top}(\mathbf{Q R} \operatorname{Exp}(\delta \boldsymbol{\phi}))\right)}{\delta \boldsymbol{\phi}} \\
& =\lim _{\delta \boldsymbol{\phi} \rightarrow 0} \frac{\log (\operatorname{Exp}(\delta \boldsymbol{\phi}))}{\delta \boldsymbol{\phi}}=\mathbf{I} .
\end{aligned}
$$

\section{ACKNOWLEDGMENT}

This work has been supported by EUROC FP7-2013-NMPICT-FOF, Loco3d ANR-16-CE33-0003, the Spanish Ministry of Economy and Competitiveness under Project EB-SLAM (DPI201789564-P), and by the Spanish State Research Agency through the Mara de Maeztu Seal of Excellence to IRI MDM-2016-0656.

\section{REFERENCES}

[1] S. Thrun and M. Montemerlo, "The graph slam algorithm with applications to large-scale mapping of urban structures." Int. Journal of Robotics Research, vol. 25, no. 5-6, 2006.

[2] M. Kaess, A. Ranganathan, and F. Dellaert, "isam: Incremental smoothing and mapping," IEEE Trans. on Robotics, 2008.

[3] D. Koller and N. Friedman, Probabilistic graphical models: principles and techniques. MIT press, 2009.

[4] T. Lupton and S. Sukkarieh, "Efficient integration of inertial observations into visual slam without initialization," in IEEE/RSJ Int. Conf. on Intelligent Robots and Systems, 2009.

[5] C. Forster, L. Carlone, F. Dellaert, and D. Scaramuzza, "Imu preintegration on manifold for efficient visual-inertial maximum-a-posteriori estimation," in Robotics: Science and Systems. Georgia Institute of Technology, 2015.

[6] S. Agarwal, K. Mierle, and Others, "Ceres solver," http://ceressolver.org.

[7] R. Kummerle, G. Grisetti, H. Strasdat, K. Konolige, and W. Burgard, " $\mathrm{g}^{2} \mathrm{o}$ : A general framework for graph optimization," in Proc. IEEE Int. Conf. Robotics Autom., Shanghai, May 2011, pp. 3607-3613.
[8] V. Ila, L. Polok, M. Solony, and P. Svoboda, "SLAM++-A highly efficient and temporally scalable incremental SLAM framework," Int. J. Robotics Res., vol. 36, no. 2, pp. 210-230, 2017.

[9] F. Dellaert and M. Kaess, "Square root SAM: Simultaneous localization and mapping via square root information smoothing," Int. J. Robotics Res., vol. 25, no. 12, pp. 1181-1204, 2006.

[10] M. Kaess, H. Johannsson, R. Roberts, V. Ila, J. J. Leonard, and F. Dellaert, "iSAM2: Incremental smoothing and mapping using the bayes tree," Int. J. Robotics Res., vol. 31, no. 2, pp. 216-235, 2011.

[11] M. Angermann, P. Robertson, T. Kemptner, and M. Khider, "A high precision reference data set for pedestrian navigation using footmounted inertial sensors," in Indoor Positioning and Indoor Navigation (IPIN), 2010 International Conference on. IEEE, 2010, pp. 1-6.

[12] InvenSense, "Imu mpu6050," https://www.invensense.com.

[13] A. Santamaria-Navarro, J. Sola, and J. Andrade-Cetto, "Highfrequency mav state estimation using low-cost inertial and optical flow measurement units," in Intelligent Robots and Systems (IROS), 2015 IEEE/RSJ International Conference on. IEEE, 2015, pp. 1864-1871.

[14] E. Foxlin, "Pedestrian tracking with shoe-mounted inertial sensors," IEEE Computer graphics and applications, vol. 25, no. 6, pp. 38-46, 2005.

[15] L. J. Hutchings, "System and method for measuring movement of objects," March 1998, uS Patent 5,724,265.

[16] L. Ojeda and J. Borenstein, "Personal dead-reckoning system for gpsdenied environments," in Safety, Security and Rescue Robotics, 2007. SSRR 2007. IEEE International Workshop on. IEEE, 2007, pp. 1-6.

[17] S. Kwanmuang, "Filtering and tracking for a pedestrian deadreckoning system," Ph.D. dissertation, University of Michigan, May 2015.

[18] P. D. Groves, G. W. Pulford, C. A. Littlefield, D. L. Nash, and C. J. Mather, "Inertial navigation versus pedestrian dead reckoning: Optimizing the integration," in Proc. ION GNSS, 2007, pp. 20432055.

[19] I. Skog, J.-O. Nilsson, D. Zachariah, and P. Händel, "Fusing the information from two navigation systems using an upper bound on their maximum spatial separation," in Indoor Positioning and Indoor Navigation (IPIN), 2012 International Conference on. IEEE, 2012, pp. 1-5.

[20] S. Sukkarieh, E. M. Nebot, and H. F. Durrant-Whyte, "A high integrity $\mathrm{imu} / \mathrm{gps}$ navigation loop for autonomous land vehicle applications,' IEEE Transactions on Robotics and Automation, vol. 15, no. 3, pp. 572-578, 1999.

[21] C. Hide, T. More, C. Hill, and J. Pinchin, "Investigating the integration of a foot-mounted imu and gnss antenna," in Ubiquitous Positioning, Indoor Navigation, and Location Based Service (UPINLBS), 2012. IEEE, 2012, pp. 1-10.

[22] W. Gao, J. Li, F. Yu, G. Zhou, C. Yu, and M. Lin, "Data fusion with two nonlinear constraints on kalman filtering," in Position, Location and Navigation Symposium-PLANS 2014, 2014 IEEE/ION. IEEE, 2014, pp. 524-528.

[23] V. Malyavej, W. Kumkeaw, and M. Aorpimai, "Indoor robot localization by rssi/imu sensor fusion," in Electrical Engineering/Electronics, Computer, Telecommunications and Information Technology (ECTICON), 2013 10th International Conference on. IEEE, 2013, pp. 1-6.

[24] A. R. J. Ruiz, F. S. Granja, J. C. P. Honorato, and J. I. G. Rosas, "Accurate pedestrian indoor navigation by tightly coupling foot-mounted imu and rfid measurements," IEEE Transactions on Instrumentation and Measurement, vol. 61, no. 1, pp. 178-189, 2012.

[25] D. Chdid, R. Oueis, H. Khoury, D. Asmar, and I. Elhajj, "Inertialvision sensor fusion for pedestrian localization," in Robotics and Biomimetics (ROBIO), 2011 IEEE International Conference on. IEEE, 2011, pp. 1695-1701

[26] D. Pierce, "Incorporation of a foot-mounted imu for multi-sensor pedestrian navigation," Ph.D. dissertation, Auburn University, 2016.

[27] M. Angermann and P. Robertson, "Footslam: Pedestrian simultaneous localization and mapping without exteroceptive sensorshitchhiking on human perception and cognition," Proceedings of the IEEE, vol. 100, no. Special Centennial Issue, pp. 1840-1848, 2012.

[28] G. S. Chirikjian, Stochastic Models, Information Theory, and Lie Groups, ser. Applied and Numerical Harmonic Analysis. Basel: Birkhäuser,, 2012, vol. 2: Analytic Methods and Modern Applications. 